\title{
R. GENZEL
}

Max-Planck-Institut für extraterrestrische Physik D-8046 Garching, F.R. Germany

\begin{abstract}
This review summarizes our current knowledge about the interstellar matter between about 1.7 and $10 \mathrm{pc}$ (a) from IRS16/SGR A*, a region which now commonly is referred to as the circum-nuclear disk (CND). I will discuss first the spatial distribution of the neutral and partially ionized, interstellar matter, then its physical parameters and excitation and, finally its dynamics. For other recent reviews of the subject I refer to the articles by Güsten (1987), Genzel (1987), Genzel and Townes (1987) and Jaffe (1987).
\end{abstract}

\section{SPATIAL DISTRIBUTION}

Figure 1 shows maps of the far-infrared continuum emission at 30,50 and $100 \mu \mathrm{m}$ of the central $4^{\prime}$ of the Galaxy (Becklin, Gatley and Werner 1982). The $30 \mu \mathrm{m}$ map is centered on IRS16/SGR A* and when measured at higher spatial resolution, closely resembles the distribution of the ionized gas in the SGR A West "mini-spiral". The mid-IR continuum most likely comes from Ly- $\alpha$ heated dust grains in the central ionized gas clumps. In contrast, the $\lambda>50 \mu \mathrm{m}$ peaks about $1.7 \mathrm{pc}$ on either side of IRS16/SGR A* and is aligned approximately along the galactic plane. Becklin, Gatley and Werner (1982) conclude from these observations that centered on the nucleus is a dust ring of inner radius about $1.7 \mathrm{pc}$ whose plane is close to the line of sight. Just inside of this ring lies some of the ionized gas. At still smaller radii, there is very little dust (and gas) except the ionized streamers discussed elsewhere in this volume (Serabyn).

This picture of a central "cavity" with a surrounding highly inclined ring or disk of dense neutral gas and dust has since been confirmed and investigated in much detail. The corresponding gas was first found in the neutral atomic oxygen fine-structure line at $63 \mu \mathrm{m}$ (Lester et al. 1981, Genzel et al. 1985), in the $2 \mu \mathrm{m} \mathrm{S}(1)$ line of vibrationally excited molecular hydrogen (Gatley et al. 1984), in the $21 \mathrm{~cm}$ transition of $\mathrm{HI}$ and in the $2.6 \mathrm{~mm} \mathrm{CO} \mathrm{J=1-0} \mathrm{rotational} \mathrm{line}$

(a) for convenience, all parameters are for a $R_{0}=10 \mathrm{kpc}$ distance to the galactic center, and have to be scaled accordingly for other values of $R_{o}$ (see Reid in this volume). 

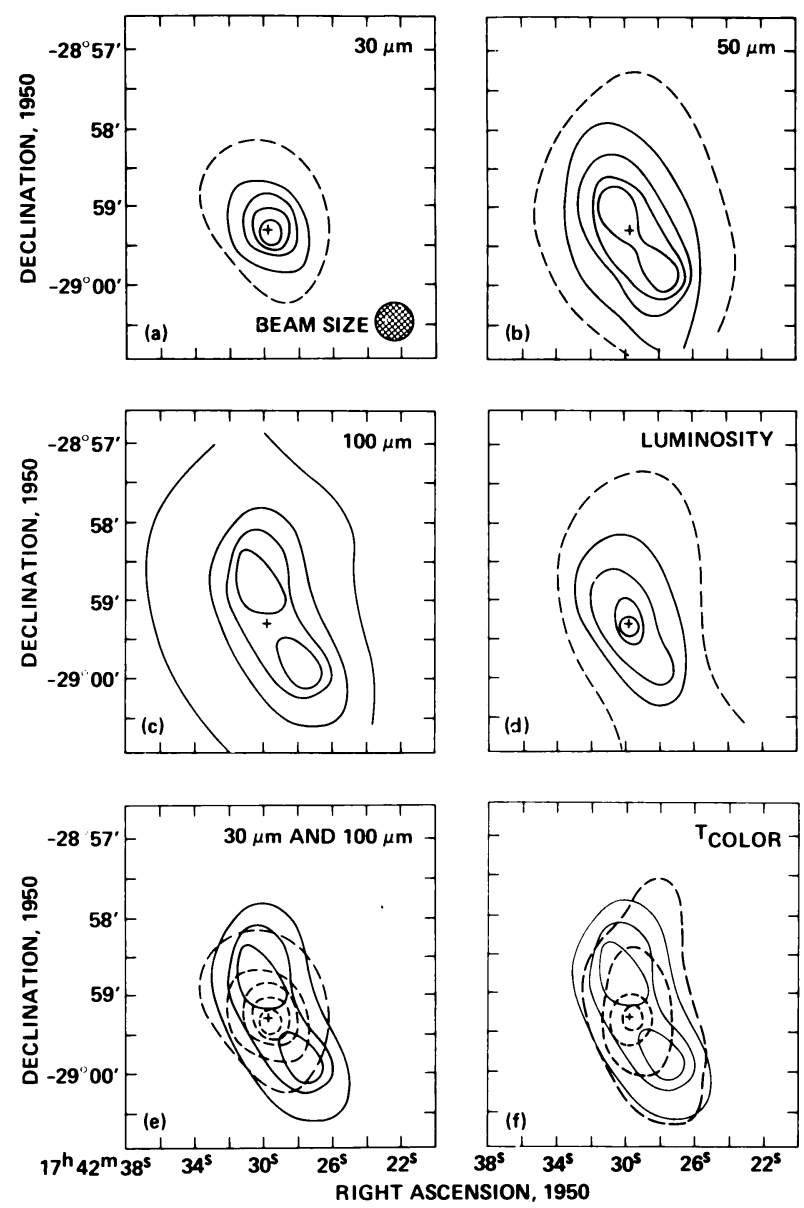

Figure 1. High angular resolution far-infrared continuum observations of the galactic center (Becklin, Gatley and Werner 1982). (a)-(c) Maps of the galactic center region are made with $30^{\text {" }}$ resolution and a common focal-plane aperture at 30,50 and $100 \mu \mathrm{m}$. The entire region within the figure was mapped at each wavelength. The cross denotes the position of the centroid of the galactic center emission at $34 \mu \mathrm{m}$ as derived from ground-based measurements. (d) Map of the 25-130 $\mu \mathrm{m}$ luminosity of the galactic center, derived from the 30,50 , and $100 \mu \mathrm{m}$ flux maps. The contour levels are $0.125,0.25,0.50,0.75$, and $1.0 \times 10^{6}$ $\mathrm{L}_{\odot}$ /beam. (e) The $30 \mu \mathrm{m}$ map (dashed) is shown superimposed upon the central contours of the $100 \mu \mathrm{m}$ map. (f) The 50-100 $\mu \mathrm{m}$ color temperature of the far-infrared emission from the galactic center, derived from the 50 and $100 \mu \mathrm{m}$ maps and assuming gray particle emission, is shown as the dashed lines superposed on the central contours of the $100 \mu \mathrm{m}$ map. From lowest to highest, the temperature contour levels are $60,70,80$, and $100 \mathrm{~K}$. 
(Liszt et al. 1983). Sandqvist (1987) notes that in retrospect some evidence for the circum-nuclear gas was already evident in his earlier lunar occultation studies of the $18 \mathrm{~cm}$ OH lines.

Recent observations at high spatial resolution now demonstrate that the inferred ring indeed exists, is essentially complete and forms the inner edge of a thin, disk-like structure extending to at least 8 pc from the center (Güsten et al. 1987, Gatley et al. 1986, Lugten et al. 1986, Kaifu et al. 1987, Serabyn et al. 1986). As an example, Figure 2 shows a velocity-integrated map of $3 \mathrm{~mm}$ HCN J=1-0 line radiation, obtained with the Hat-Creek interferometer at 4" resolution, superposed on a VLA $6 \mathrm{~cm}$ radio continuum map (Lo and Claussen 1983). The ring is very clumpy and inclined by about 60 to $70^{\circ}$ with respect to the line of sight. Inside its sharp inner edge, there is a thin ring of ionized gas on its western side, but very little other interstellar gas. The mean density of neutral gas at $R<1.7 \mathrm{pc}$ is at least a factor of 10 lower than in the neutral ring itself.

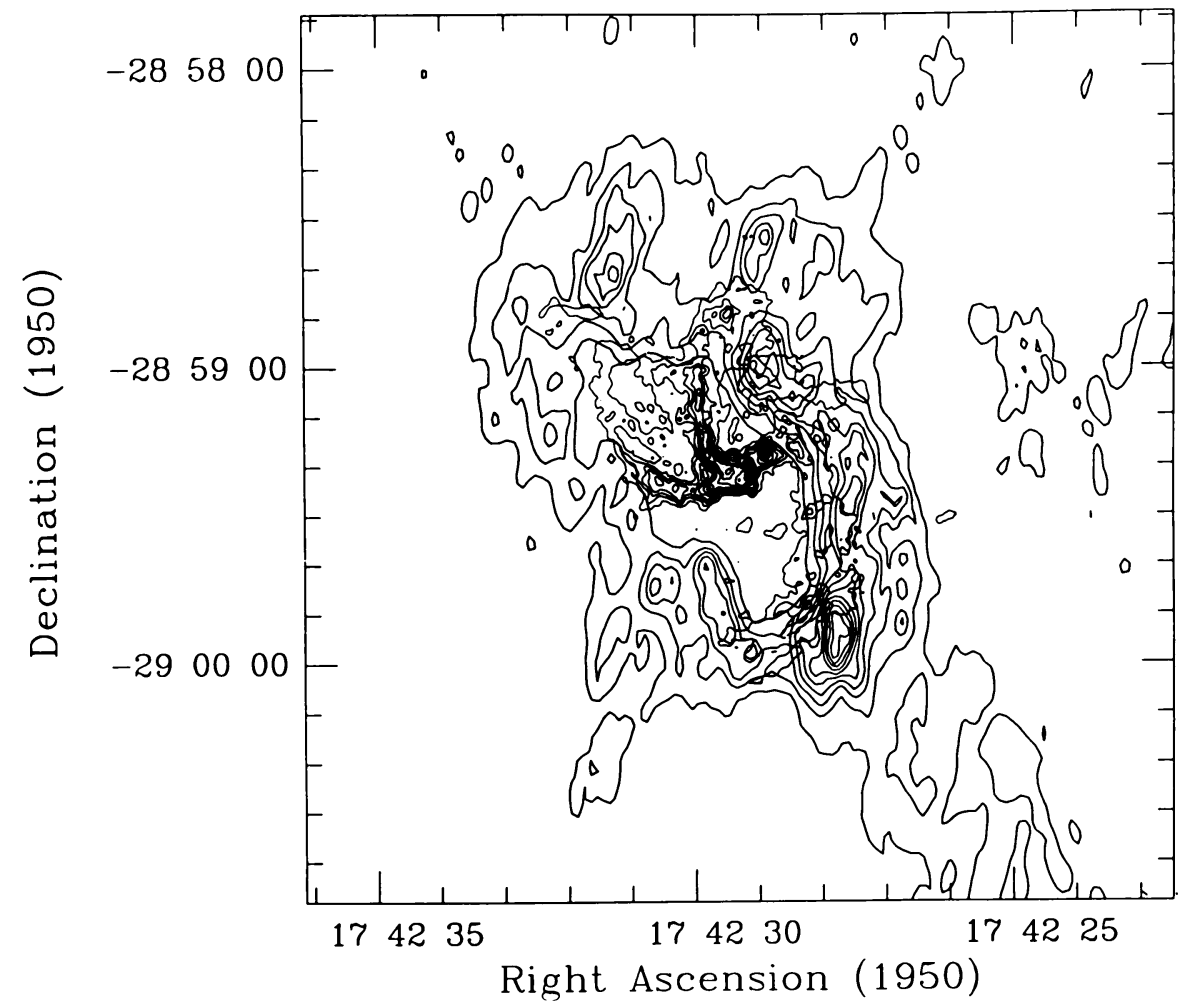

Figure 2. A 3-mm HCN J=1-0 aperture synthesis map at 4" resolution (MEM; Wright et al. 1987) combined with the $6-\mathrm{cm}$ radio continuum map from Lo and Claussen (1983); 1" resolution). 
At the inner edge of the CND warm and hot molecular gas, sampled by the $3 \mathrm{~mm} \mathrm{HCN}$ and $2 \mu \mathrm{m} \mathrm{H} \mathrm{H}_{2}$ line emission, is coextensive with warm dust grains and atomic gas observed by far-infrared [OI] and [CII] fine structure line emission. Cooler molecular and atomic gas is found at larger radii, indicative of a composition and excitation gradient (see below). This is shown in Figure 3 which gives radial cuts of the different tracers along the galactic plane and centered on IRS16/SGR $A^{*}$.

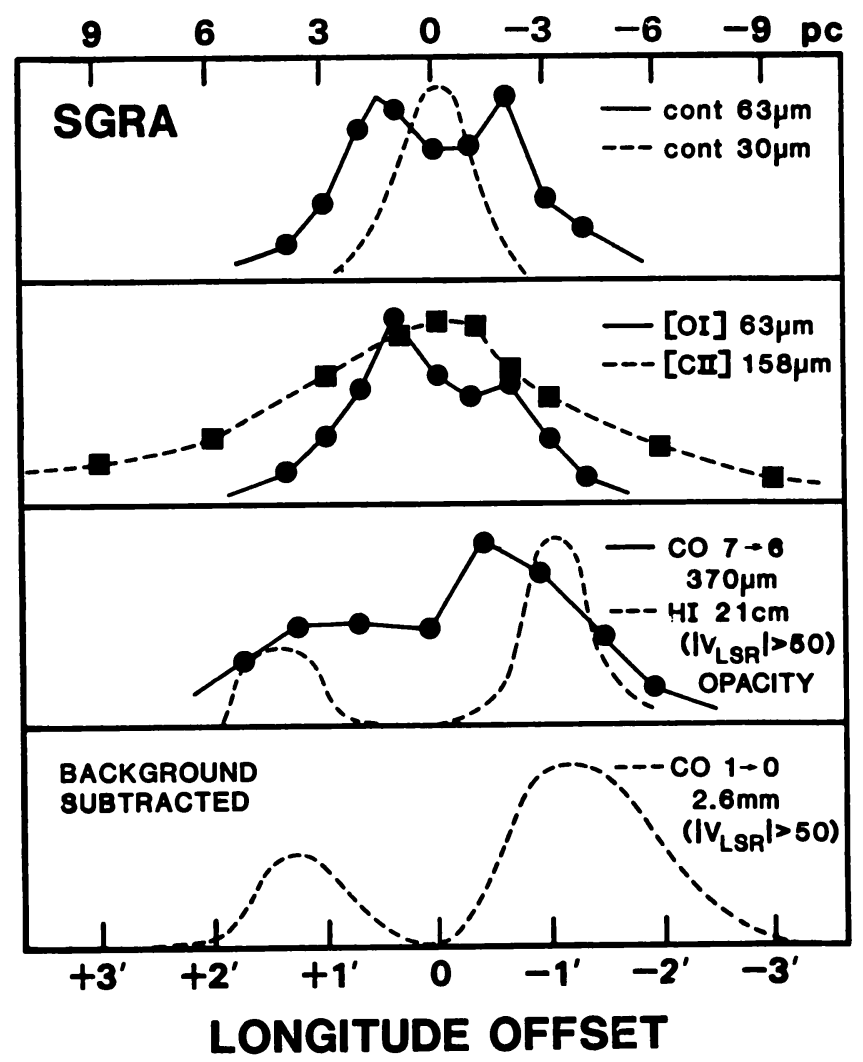

Figure 3. Cuts of various tracers of dust, atomic and molecular gas through the CND (along the galactic plane). Offsets are relative to SgrA*. The FIR continuum (top: Becklin et al. 1982), [OI] and [CII] emission (second from top: Genzel et al. 1985, Lugten et al. 1986), CO 7-6 (Harris et al. 1985) and HI $21 \mathrm{~cm}$ (Liszt et al. 1983) and CO 1-0 (bottom: Liszt et al. 1983, Serabyn et al. 1986) data have spatial resolution of typically $30^{\prime \prime}$ to $1^{\prime}$. 


\section{PHYSICAL CONDITIONS}

Figure 4 (from Lugten et al. 1987) shows estimates of density and kinetic temperature of the molecular gas as a function of position offset from the center, obtained from a comparison of observed farinfrared, submm and $\mathrm{mm}$ CO line ratios with collisional excitation calculations (see Harris et al. 1985). The physical parameters of the warm atomic gas are similar to those of the molecular material (Genzel et al. 1985). The measurements clearly show that the atomic and molecular gas in the circumnuclear disk is unusuallly warm and dense and that excitation, energy density and gas pressure fall rapidly with increasing radius. The gas kinetic temperature of most of the gas is in excess of a few hundred $K$, that is, definitely higher than the dust temperature ( 50 to $70 \mathrm{~K}$, Becklin et al. 1982). There is also a range of densities and temperatures at any given point in the disk. For example, measurements of several CS rotational lines (Güsten et al. this volume) indicate hydrogen densities of 2 to $4 \times 10^{5} \mathrm{~cm}^{-3}$, about ten times higher than the densities of the $C O$ emitting region, and probably indicative of densities in the clump cores. The $2 \mu \mathrm{m} \mathrm{H}_{2}$ emission lines, on the other hand, come from gas of temperature near 1000 to $2000 \mathrm{~K}$ (Gatley et al. 1984). Table 1 gives the parameters for a typical clump in the CND.

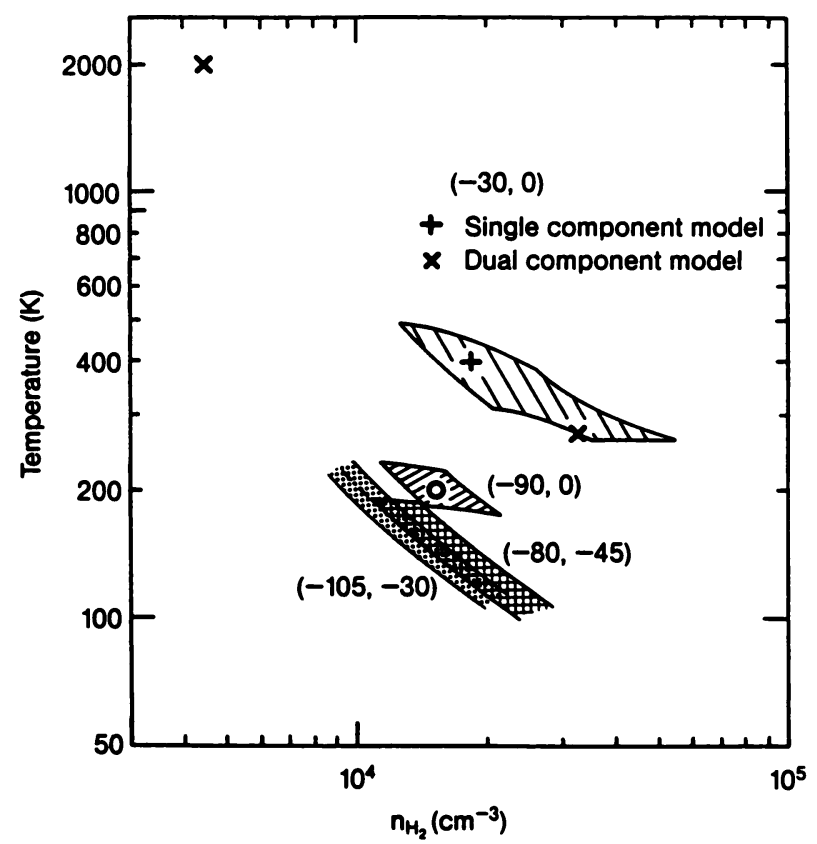

Figure 4. Density and temperature of molecular gas at different positions in the CND, as estimated from collisional excitation models of $\mathrm{mm}$, submm and far-infrared CO lines (Lugten et al. 1987). The offsets are galactic longitude and latitude relative to SgrA*. 
One interesting characteristic of the CND is its low volume filling factor; only 5 to 108 of the volume is actually filled with gas. Furthermore, there is as yet very little evidence for a substantial intercloud medium. From a comparison of the 1-0 and 2-1 CO measurements by Serabyn et al. (1986) and Lo (1986), and the 7-6 Co data by Harris et al. (1985) the column density of any cold ( $T \ll 100 \mathrm{~K}$ ) inter-cloud (as well as intra-clump) gas is 0.3 or less of the column density of warm gas associated with the clumps. Any hot intercloud gas would have to have a column density less than 108 of the warm gas. Mezger et al. (1988) find submillimeter dust emission from the CND which they interpret to come from cold $\left(\mathrm{T}_{\boldsymbol{r}}=10\right.$ to $20 \mathrm{~K}$ ) dust within the clumps. Given the apparently large pressure contrast between clumps and their environment an important question is how the clumps can be maintained over any length of time.

TABLE 1. Typical parameters of a clump in the CND.

$$
\begin{aligned}
& \text { Diameter : } 0.2 \mathrm{pc}(4 ") \\
& \text { Velocity dispersion (FWHM) : } 30 \text { to } 50 \mathrm{~km} \mathrm{~s}^{-1} \\
& \mathrm{n}\left(\mathrm{H}_{2}\right): 3 \times 10^{4} \text { (overall) } \rightarrow 3 \times 10^{5} \text { (core) } \mathrm{cm}^{-3} \\
& \mathrm{~N}\left(\mathrm{H} \mathrm{H}_{2}\right): 5 \times 10^{22}\left(\mathrm{~A}_{\mathrm{v}}>30\right) \\
& \text { Mass : } 30 \mathrm{M}_{\odot} \\
& \text { Atomic to molecular mass fraction : } 0.1-0.2 \\
& \mathrm{~T}_{\text {gas }}: 100-500 \mathrm{~K}(\mathrm{CO}, \mathrm{HCN}, \text { OI/CII) } \\
& \quad 1000-2000 \mathrm{~K}\left(\mathrm{H}_{2}\right) \\
& \mathrm{T}_{\text {dust }}: 50 \text { to } 90 \mathrm{~K}
\end{aligned}
$$

Another important characteristic of the CND is the large luminosity of the far-infrared and submillimeter line radiation (Genzel et al. 1985, Harris et al. 1985) which is a direct consequence of the high gas temperatures and densities. About $2 \times 10^{4} \mathrm{I}_{\odot}$ emerges in the far-infrared fine structure lines of [OI], [CII] and [SiII] (Genzel et al. 1985, Lugten et al. 1986, Graf et al. 1988), about the same amount is contained in the rotational lines of CO (Lugten et al. 1987, Harris et al. 1985) and about $10^{3} \mathrm{~L}_{\odot}$ is radiated in the near-infrared $\mathrm{H}_{2}$ lines (Gatley et al. 1984). Hence, the total line radiation from 
excited atomic and molecular gas is about 18 of the bolometric luminosity of SGR A, requiring effective heating mechanisms. Another observational constraint is that the line emission of excited gas, although brightest near the inner edge of the CND, typically extends 1 to $3 \mathrm{pc}$ into the CND (Genzel et al. 1985, Lugten et al. 1986, Harris et al. 1985). Note that this is also the case for the $2 \mu \mathrm{m}$ molecular hydrogen emission (Krabbe et al. 1988) which thus cannot just come from a thin shock front at the inner edge of the disk. The gas heating thus must either come from distributed sources throughout the disk or, if it originates in the center, it must penetrate sufficiently far into the disk. The latter mechanism probably explains the far-infrared atomic lines. UV radiation from the central 2 pc penetrates efficiently into the disk because of its clumpiness, and creates photodissociation regions at the surfaces of clumps. The main mechanism may be photoelectric heating (Harris et al. 1985, Tielens and Hollenbach 1985). Heating by the center's UV radiation may also be important for the molecular gas, although shock excitation by clump-clump collisions due to the disk's large local turbulence (Harris et al. 1985 and below) may be more likely. Shock excitation by a wind from the center could also explain the $2 \mu \mathrm{m} \mathrm{H}_{2}$ lines (Gatley et al. 1984), but not the much more intense far-infared and submillimeter $\mathrm{CO}$ emission.

Table 1 indicates that the $\mathrm{H}_{2}$ column densities through the clumps of the CND are about $5 \times 10^{22}$ or $A_{v}=30$ for the standard interstellar gas -to-dust conversion ratio. Hence, one ought to expect a significant impact of the CND gas distribution on the appearance of near-infrared maps of the central cluster.

This conjecture is borne out by recent data. Figure 5 shows a $\mathrm{H}$-band $(1.6 \mu \mathrm{m})$ map of the central $70^{\prime \prime}$ taken with the UKIRT IRCAM (Aspin et al. 1988), superposed on the Hat Creek HCN map shown earlier. The infrared emission (mostly from $\mathrm{K}$ and $\mathrm{M}$ giants, see Sellgren in this volume) drops markedly at the ring's location. The drop is sharpest at the southwestern edge of the ring, indicating that this side is in front of the galactic center. From the measured drop $(\Delta H=1$ to 2) and the simplifying assumption that the thickness of the CND is small (see below) compared to the scale height of the stars, so that at maximum only half of the cluster is behind the CND, we infer $A_{v}>20$ through the disk, consistent with the above expectation. There are several interesting consequences. First, because the near-infrared emission is distorted locally by extinction, estimates of the cluster's mass distribution from its near-infrared light distribution have to be considered with caution even for the central few pc (see also the discussion by Lebofsky (1979)). Second, any estimate of a core radius (cf. Rieke and Lebofksy 1987, and Allen 1987) not taking into account the disk's obscuration is strictly only a lower limit. Third, the reason for the relatively low $\left(A_{v}=27\right)$ and spatially constant extinction of the central 2 pc radius region is the presence of a cavity local to the galactic center region. 


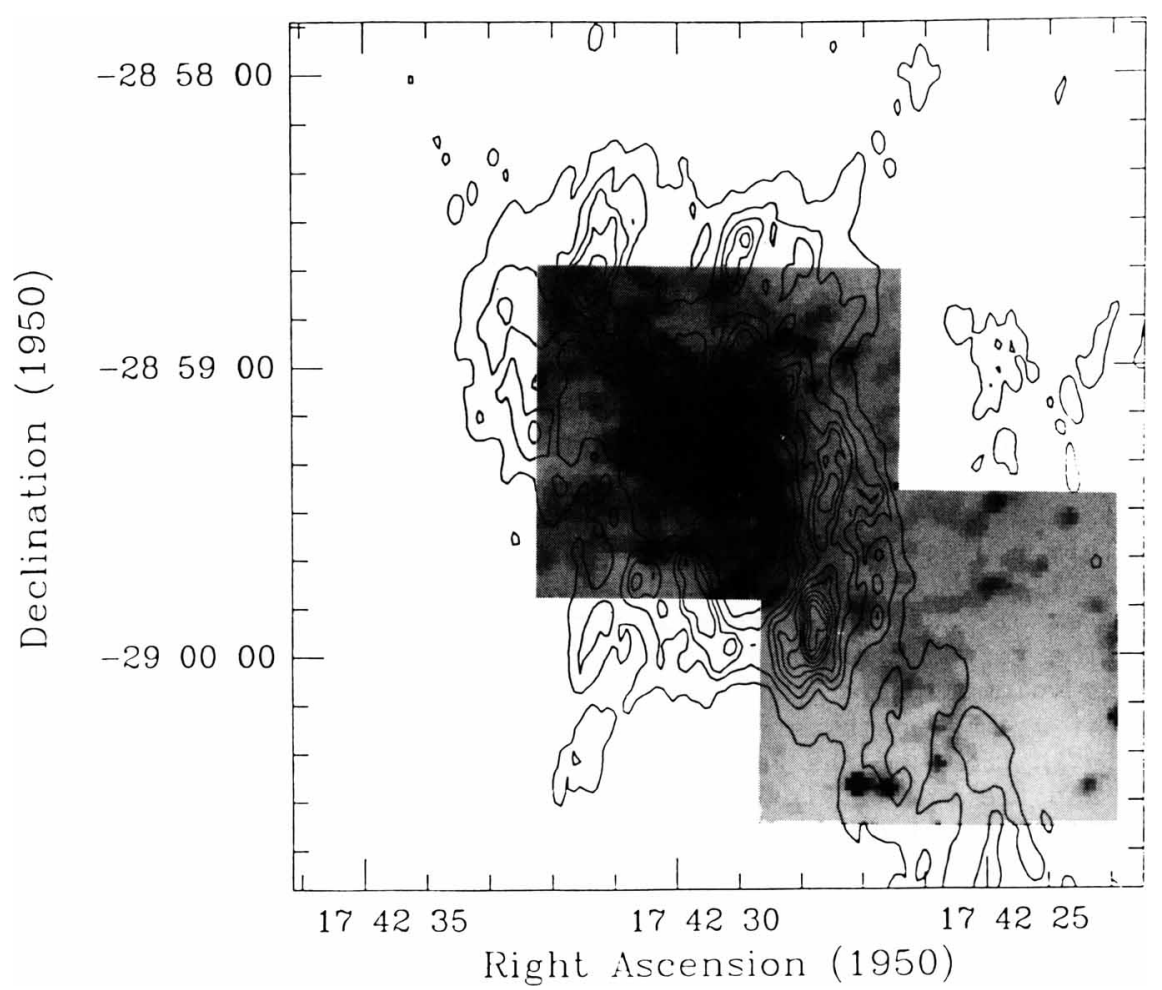

Figure 5. Overlay of a composite $1.6 \mu \mathrm{m}$ (H-band) image on the Hat Creek HCN map of Fig. 1. The infrared data, taken with the UKIRT infrared camera (IRCAM), are from the work by Aspin et al. (1988). 


\section{KINEMATICS AND DYNAMICS}

The available infrared and microwave spectroscopic measurements give detailed information on the neutral gas dynamics. The dominant largescale motion of the majority of the neutral gas is rotation about the center. Several investigations find that any systematic, large-scale radial motion of the CND as a whole must be less than about $30 \mathrm{~km} / \mathrm{s}$, while a few clouds in the disk show strong deviations from circular

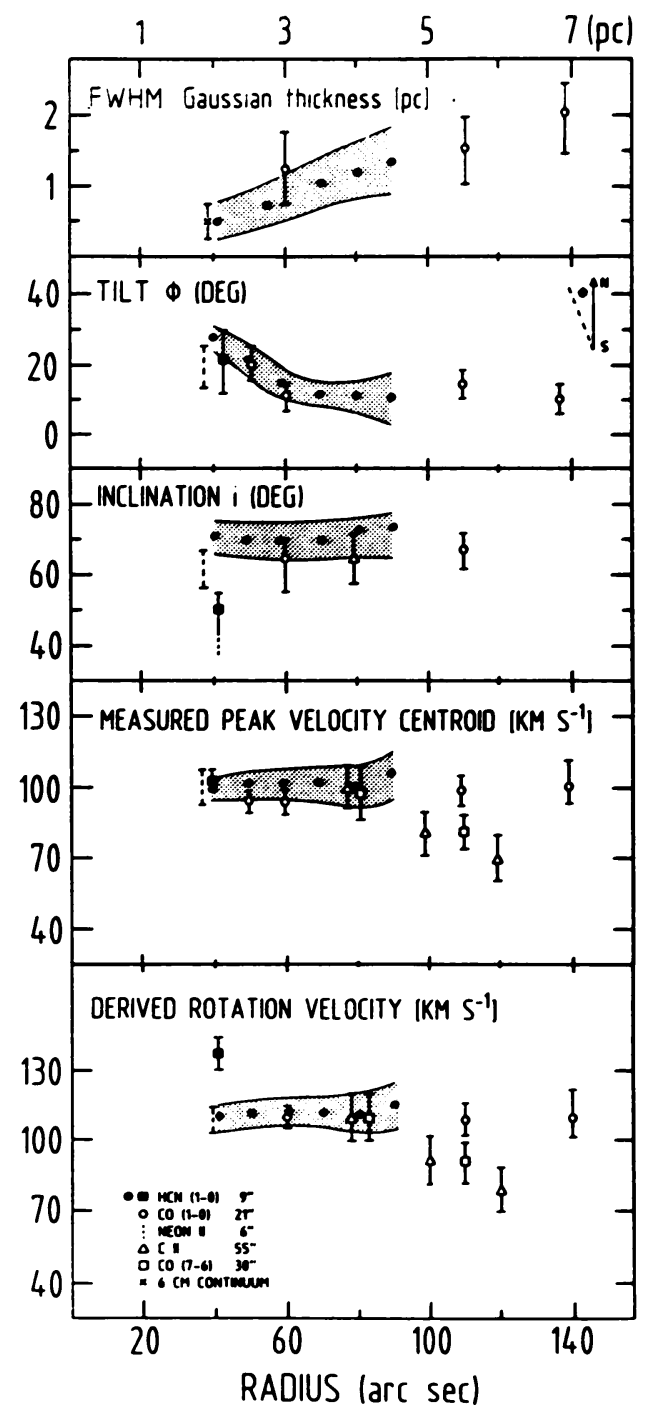

Figure 6. Parameters of the neutral circumnuclear disk as derived from a number of measure ments discussed in the text (from Güsten et al. 1987). 
motion (Güsten et al. 1987, Lugten et al. 1986, Serabyn et al. 1986, see also Sandqvist et al. this volume). On the other hand, Gatley et a1. (1986) and Kaifu et al. (1987) conclude systematic radial motions as large as $50 \mathrm{~km} / \mathrm{s}$ are characteristic of the inner $2 \mathrm{pc}$ ring. The derived rotation velocity as a function of distance from the center, corrected for inclination $\left(70^{\circ}\right)$, is approximately constant between 2 and $4 \mathrm{pc}$ ( $\mathrm{v}_{\text {rot }}=110 \mathrm{~km} / \mathrm{s}$, Fig. 6). Beyond $4 \mathrm{pc}$ the velocity field is more complex, mainly due to the appearance of lower velocity gas in the high excitation [CII], CO 3-2 and 7-6 and CS transitions. It is presently not clear whether the higher or the lower velocity gas is represenatative of the disk's rotation. A likely explanation of the lower velocity gas (see Lugten et al. this volume) is the presence of dense, warm material off the major axis of the disk.

While a circulating disk is a good first-order description of the neutral gas dynamics, the rotation pattern is strongly disturbed. Large local line widths everywhere in the CND $\left(\Delta \mathrm{v}_{\mathrm{FWHM}^{-}}=50 \mathrm{~km} / \mathrm{s}\right.$ in a $4 "$ beam) indicate turbulence or other large, local perturbations in the circular motion. As mentioned, there are clouds which definitely show strong $(50 \mathrm{~km} / \mathrm{s})$ non-circular motions, such as a bright cloud at the inner edge of the ring due west of IRS16/SGR A* (see Güsten et al. 1987, Gatley et al. 1986, Serabyn et al. 1986), or an unusual streamer seen in the $18 \mathrm{~cm} \mathrm{OH}$ transitions apparently trailing from the ring toward SGR A* (Sandqvist et al. this volume).

Furthermore, the spatial distribution and velocity field of the $\mathrm{HCN}$ gas at the inner edge of the disk indicate that not all of the material is in one plane, but rather that the inner disk is somewhat warped, or that it consists of a number of separate gas streamers in slightly different orientations (Güsten et al. 1987). Finally, the disk's position angle on the sky appears to change with distance from the center (Fig. 6). The plane of rotation is within $5^{\circ}$ of the Galactic plane at $2 \mathrm{pc}$, but is tilted by about $25^{\circ}$ relative to the plane at radii of 3 to 5 pc.

The large local turbulence makes up about 10 to 158 of the rotational energy and may support the thickness of the CND. The disk's FWHM thickness increases from about $0.5 \mathrm{pc}$ at $2 \mathrm{pc}$ to about $2 \mathrm{pc}$ at 7 pc, approximately consistent with hydrostatic equilibrium (Fig. 6).

There appears to be a strong magnetic field in the plane of the disk. Werner et al.(1988) and Werner and Davidson (this book) conclude from polarization measurements of the far-infrared continuum that the field is predominantly toroidal. Since the degree of polarization is approximately constant from the inner edge to several pc radius, the magnetic field may also have a significant random component. If the magnetic field energy is appproximately equal to the turbulent energy, the field strength in the disk is about $10 \mathrm{mG}$ (Werner et al. 1988). Two important questions emerge. Does the magnetic field create and hold together the clumps, and is the disk's toroidal field connected with the poloidal field inferred from radio polarization studies in the halo of SGR A (see Yusef-Zadeh this volume)?

The present distribution and physical parameters of the CND cannot represent static equilibrium. Although the neutral gas clouds are subject to tidal disruption and dispersal because of substantial velo- 
city differentials, the disk is nevertheless clumpy and has a well defined, sharp inner edge. Radiation pressure, thermal pressure from the HII region, or hot gas in the cavity are not sufficient to maintain the sharp edge. The apparent warping also cannot be maintained in steady state. The time scale for dispersal of the clumpy clouds or the warping is no more than a few rotation periods, or a few $10^{5} \mathrm{yr}$. The time scale for dissipation of the disk's turbulent energy ( $2 \times 1050$ ergs) by shock cooling, as estimated from gas mass, turbulent velocity dispersion and the observed infrared and submillimeter line cooling also is no longer than about $10^{5} \mathrm{yr}$. Finally, unless there are (specially configured magnetic) forces pressurizing the central cavity, the lower velocity gas at the inner edge will fall inward, eliminating the sharp density discontinuity on a time scale of not much longer than $10^{5}$ yr.

A possible dynamical equilibrium of the CND might be conversion of rotational into turbulent energy, possibly aided by magnetic 'friction', leading to a slow contraction of the disk. In this scenario (Güsten et al. 1987), the mass accretion necessary to maintain the present line radiation is about $10^{-2} \mathrm{M} \odot / \mathrm{yr}$. Over a period of a few million years, however, the central cavity would fill in unless there is a mechanism for its removal, and mass has to be replenished from farther out. A possible source for material flowing into the center may be the +20 and $+50 \mathrm{~km} / \mathrm{s}$ molecular clouds near the center which are very likely within 10 to 30 pc of SGR A West (Güsten et al. 1987, Mezger et al. 1988, Lugten et al. 1986, Genzel et al. this volume). Ho et al. (this volume) reports the discovery of a linear streamer of gas leading from the $+20 \mathrm{~km} / \mathrm{s}$ cloud to the south-eastern side of the inner 2 pc ring, possibly representing such a physical connection between the CND and the much more massive clouds at $R>10 \mathrm{pc}$. Another significant influx of gas into this region may be mass loss from asymptotic giant branch stars as measured by IRAS and $18 \mathrm{~cm} \mathrm{OH}$ maser emission (Habing 1987). An alternative scenario is an explosion (see connection to SGRA East: Mezger et al. (this volume and 1988)) or a burst of mass outflow with a required energy of $10^{51}$ ergs no longer than $10^{5}$ yr ago. Such temporary, intense activity could also explain the central cavity.

In place of a summary, Figure 7 shows schematically a possible scenario of the CND, assembled from the data discussed in this review. A clumpy, turbulent, irregular disk surrounds and rotates about the nucleus of our Galaxy. Clump-clump collisions, UV radiation and possible mass outflow from the central cavity compress and heat up the gas. As proposed originally by Lo and Claussen (1983), the western arc of the ionized gas (see Serabyn and Lacy 1985) is the photoionized inner edge of the disk, and the other ionized gas streamers (such as the northern and eastern arms) represent infalling clouds which have lost angular momentum as a result of a collision in the CND and are ionized and tidally stretched during their fall into the cavity. About the eventual fate of this gas, the origin and lifetime of the cavity, and the connection to the central energy sources, much more needs to be learned in the next few years. 


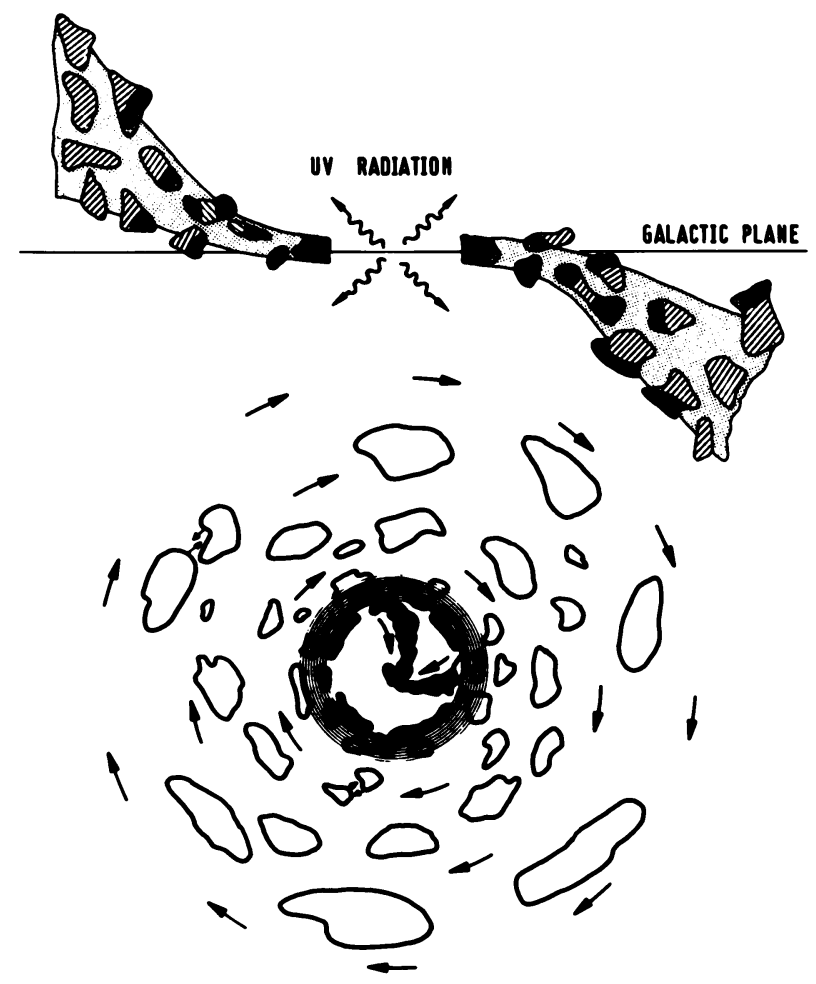

Figure 7. Schematic model of the circum-nuclear disk and ionized gas streamers in the central $6 \mathrm{pc}$ of the Galaxy. The top graph shows the clumpiness, excitation structure and bending with radius from an edge-on view. Black indicates ionized gas, heavy shading warm atomic gas, and shading dense molecular gas. Less dense interclump gas is indicated by the light shaded zones. The bottom graph is a face-on view showing rotation and turbulence, the hot shocked inner zone, the ionized inner edge and the infalling streamers. Black again is ionized gas and the circular shaded region marks the inner ring where hot $\mathrm{H}_{2}$ is found.

\section{ACKNOWLEDGEMENTS}

I thank my colleagues R. Güsten, A. Harris, D. Jaffe, J. Lacy, J. Lugten, E. Serabyn, J. Stutzki and C.H. Townes for their help and illuminating discussions. Much of this summary is also contained in the recent Annual Reviews Article by C.H. Townes and myself (Genzel and Townes 1987). I thank C. Aspin, I. McLean, A. Sandqvist and H. Zinnecker for letting me use data prior to publication. 


\section{REFERENCES}

Allen, D.A. 1987, see Backer (1987), p.1..

Aspin, C., Genzel, R., McLean, I. and Zinnecker, H. 1988, in prep.

Backer, D.C. 1987, The Galactic Center. Proceedings of the AIP Conference No. 155 in Honor of Charles H. Townes, Am. Inst. Phys., New York.

Becklin, E.E., Gatley, I., Werner, M.W. 1982. Ap.J. 258:134.

Gatley, I. Jones, T.J., Hyland, A.R., Beattie, D.H., Lee, T.J. 1984, MNRAS 210:565.

Gatley, I., Jones, T.J., Hyland, A.R., Wade, R., Geballe, T.R., Krisciunas, K.L. 1986. MNRAS 222:299.

Genze1, R. 1987. in R. Carswe11, G. Gilmore (eds.), The Galaxy, Proc. NATO Summer School, Reidel, Dordrecht, pp. 51.

Genzel, R., Watson, D.M., Crawford, M.K., Townes, C.H. 1985, Ap.J. $297: 766$.

Genzel, R. and Townes, C.H. 1987, Ann.Rev.Astr.Ap. 25:377-423.

Graf, P., Herter,T., Gull,G.E. and Houck,J.R. 1988, Ap.J., in press.

Güsten, R. 1987, see Backer (1987), p. 19.

Güsten, R., Genze1, R., Wright, M.C.H., Jaffe, D.T., Stutzki, J., Harris, A.I. 1987, Ap.J. 318:124.

Habing, H.J. 1987, in R. Carswell, G. Gilmore (eds.), The Galaxy, Proc. NATO Summer School, Reidel, Dordrecht, p.

Harris, A.I., Jaffe, D.T., Silber, M., Genzel, R. 1985, Ap.J.Lett., $294:$ L93.

Jaffe, D.T. 1987, in M.P. Ulmer (ed.), Proceedings $13^{\text {th }}$ Texas Symposium, World Scientific (Singapore), p. 366.

Kaifu, N., Hayashi, M., Inatami, J., Gatley, I. 1987, see Backer (1987), p. 106.

Krabbe, A. et al. 1988, in prep.

Lebofsky, M.J. 1979, Astron.J. 84:324.

Lester, D.F., Werner, M.W., Storey, I.W.V., Watson, D.M., Townes, C.H. 1981, Ap.J.Lett. 248:L109.

Liszt, H.S., van der Hulst, J.M., Burton, W.B., Ondrechen, M.P. 1983, Astron. Astrophys. $126: 341$.

Lo, K.Y. 1986, Science 233:1394.

Lo,K.Y., Claussen, M.J. 1983, Nature 306:647.

Lugten, J.B., Genzel, R., Crawford, M.K. and Townes, C.H. 1986, Ap.J. $306: 691$.

Lugten, J.B., Harris, A.I., Stacey, G.J., Genzel, R., Townes, C.H. 1987, see Backer (1987), p. 118.

Mezger, P.G., Zylka, R., Salter, C.J., Wink, J.E., Chini, R., Kreysa, E. and Tuffs, R. 1988, Astr.Ap., in press.

Rieke, G.H. and Lebofsky, M.J. 1987, see Backer (1987), p. 91.

Sandqvist, Aa. 1987, see Backer (1987), p. 168.

Serabyn, E., Lacy, J.H. 1985, Ap.J. 293:445.

Serabyn, E., Güsten, R., Wink, J.E., Walmsley, C.M., Zylka, R. 1986, Astron. Astrophys. $169: 85$.

Tielens, , A.G.G.M. and Hollenbach, D. 1985, Ap.J. 291:772.

Werner, M.W. , Davidson, J.A., Morris, M., Novak, G., Platt, S.R. and Hildebrand, R.H. 1988, Ap.J., in press.

Wright, M.C.H., Genzel, R., Güsten, R., Jaffe, D.T. 1987, see Backer 1987 , p. 133. 